\title{
A Decision Making Model for the Taiwanese Shipping Logistics Company in China to Select the Container Distribution Center Location
}

\author{
Chien-Chang Chou \\ Department of Shipping Technology, National Kaohsiung Marine University \\ Kaohsiung, Taiwan, Republic of China \\ chiench1@ms58.hinet.net
}

\begin{abstract}
The purpose of this paper is to propose a decision making model for the Taiwanese shipping logistics company in China to select the best container distribution center location. The representation of multiplication operation on fuzzy numbers is useful for the decision makers to solve the fuzzy multiple criteria decision making problems of container distribution center location selection. In the past, few papers discussed the representation of multiplication operation on multiple fuzzy numbers. Thus this paper first compute and obtain the representation of multiplication operation on multiple fuzzy numbers. Based on this representation, the decision maker can rank quickly the ordering of each alternative location and then select easily the best one. Finally, the representation of multiplication operation on multiple fuzzy numbers is applied to solve the fuzzy multiple criteria decision making problem of container distribution center location selection in China.
\end{abstract}

\section{Introduction}

In the recent years, a lot of Taiwanese business companies went to China to invest the shipping logistics industry, manufacturing industry, civil engineering industry, banking industry and so on. It is an important and difficult for the Taiwanese business company to select a best location for building the container logistics center or the manufacturing factory when beginning the investment planning. Thus, the purpose of this paper is to propose a decision making model for the Taiwanese shipping logistics company in China to select the best container distribution center location.

The selection of location is one of the most important decision issues for the decision makers of business companies or industrial organizations. Many precisionbased methods for location selection have been developed. Dahlberg and May [7] utilized the simplex method to determine the optimal location of energy facilities. Tompkins and White [20] introduced a method that used the preference theory to assign weights to subjective factors by making all possible pairwise comparisons between factors. Spohrer and Kmak [18] proposed a weight factor analysis method to integrate the quantitative data and qualitative rating to choose a plant location from numerous alternatives. Stevenson [19] proposed a cost-volume analysis method to select the best plant location. Multiple criteria decision-making methods were 
provided to deal with the problem of ranking and selecting locations under multiple criteria $[11,17]$. All the methods stated above are based on the concept of accurate measure and crisp evaluation.

Generally, the selection of a best location for business companies and industrial organizations from two or more alternatives locations on the basis of two or more factors is a multiple criteria decision-making problem. Under many situations, the values for the qualitative criteria are often imprecisely defined for the decision maker. It is not easy to precisely quantify the rating of each alternative location and the precision-based methods as stated above are not adequate to deal with the location selection problem [2, 13]. Since human judgments including preference are often vague and can not estimate his preference with an exact numerical value. A more realistic way may be to use linguistic terms to describe the desired value and important weight of criteria, e.g. "very low", "low", "fair", "high", "very high", etc $[1,24]$. Due to this type of existing fuzziness in the location selection process, fuzzy set theory is an appropriate method for deal with uncertainty and the subjective evaluation data can be more adequately expressed in fuzzy linguistic variables $[2,10$, $13,21,22,24]$.

The concept of fuzzy sets, which was introduced by Zadeh [23], led to the definition of the fuzzy number and its implementation in fuzzy control and approximate reasoning problems. The basic arithmetic structures for fuzzy numbers were developed by Mizumoto and Tanaka [15], Nahmias [16], Dubois and Prade [9], Li [12], Ma et al. [14] and Delgado et al. [8]. The arithmetic operation was established either by the extension principle or by observing the fuzzy number as a collection of $\alpha$-levels. Unfortunately, although there are many arithmetical operation approaches, none of these approaches focuses on the representation of multiplication operation on multiple fuzzy numbers. The representation of multiplication operation on multiple fuzzy numbers is useful for the decision makers to solve the fuzzy multiple criteria decision making problems of location selection. Chou [4] proposed the canonical representation of multiplication operation on two triangular fuzzy numbers by the Graded Multiple Integration Representation Method. Chou [5] further proposed the canonical representation of multiplication operation on two trapezoidal fuzzy numbers by the Inverse Function Arithmetic Representation Method and this representation is applied to a numerical example. Based on the Inverse Function Arithmetic Representation Method, Chou [6] further proposed the representation of multiplication operation on three fuzzy numbers and this representation is applied to solve the marine transshipment container port selection problems. In this paper, we will further propose the representation of multiplication operation on multiple triangular fuzzy numbers.

The rest of this paper is organized as follows. In section 2, we use the Inverse Function Arithmetic Representation Method for multiplication operation on multiple fuzzy numbers. Based on the Inverse Function Arithmetic Representation Method, it is easy to interpret clearly and compute quickly the canonical representation of multiplication operation with the membership functions of fuzzy numbers. Finally, The representation of multiplication operation on multiple fuzzy numbers proposed in this paper is applied to solve the fuzzy multiple criteria decision problem of container distribution center location selection of Taiwanese shipping logistics company in China in section 3 followed by conclusion in section 4 . 


\section{The Representation of Multiplication on Multiple Fuzzy Numbers}

In this section, we first introduce briefly the graded mean integration representation method for presenting the representation of one fuzzy number. Based on the graded mean integration representation method, we further propose the Inverse Function Arithmetic Representation Method for presenting the representation of multiplication operation on multiple fuzzy numbers.

\subsection{The Graded Mean Integration Representation Method}

Chen and Hsieh [3] proposed the graded mean integration representation method of fuzzy numbers based on the integral value of graded mean h-level of generalized fuzzy number. Here we describe the meaning as follows.

Suppose $A=(c, a, b, d)$ is a fuzzy number. The membership function of $A$ is

$$
f_{A}(x)=\left\{\begin{array}{cc}
\frac{(x-c)}{(a-c)}, & c \leq x \leq a \\
1, & a \leq x \leq b \\
\frac{(x-d)}{(b-d)}, & b \leq x \leq d \\
0, & \text { otherwise }
\end{array}\right.
$$

Since $\quad L_{A}(x)=\frac{(x-c)}{(a-c)}, \quad c \leq x \leq a, \quad R_{A}(x)=\frac{(x-d)}{(b-d)}, \quad b \leq x \leq d$,

and $\quad \mathrm{L}^{-1}{ }_{\mathrm{A}}(\mathrm{h})=\mathrm{c}+(\mathrm{a}-\mathrm{c}) \mathrm{h}, \quad 0 \leq \mathrm{h} \leq 1, \quad \mathrm{R}^{-1}{ }_{\mathrm{A}}(\mathrm{h})=\mathrm{d}+(\mathrm{b}-\mathrm{d}) \mathrm{h}, \quad 0 \leq \mathrm{h} \leq 1$.

$L_{A}(x)$ and $R_{A}(x)$ are the function $L$ and the function $R$ of fuzzy number $A$, respectively. $L_{A}^{-1}(h)$ and $R_{A}^{-1}(h)$ are the inverse functions of function $L_{A}(x)$ and function $\mathrm{R}_{\mathrm{A}}(\mathrm{x})$ at h-level, respectively. Then the graded mean h-level value of the fuzzy number $A$ is $h\left[\mathrm{~L}^{-1}{ }_{A}(h)+R^{-1}{ }_{A}(h)\right] / 2$. The graded mean integration representation of $\mathrm{A}$ is

$$
\begin{aligned}
\mathrm{P}(\mathrm{A}) & =\int_{0}^{1} \frac{\mathrm{h}\left[\mathrm{L}^{-1}(\mathrm{~h})+\mathrm{R}^{-1}(\mathrm{~h})\right]}{2} \mathrm{dh} / \int_{0}^{1} \mathrm{hdh} \\
& =\int_{0}^{1} \frac{\mathrm{h}[\mathrm{c}+(\mathrm{a}-\mathrm{c}) \mathrm{h}+\mathrm{d}+(\mathrm{b}-\mathrm{d}) \mathrm{h}]}{2} \mathrm{dh} / \int_{0}^{1} \mathrm{hdh} \\
& =\frac{1}{6}(\mathrm{c}+2 \mathrm{a}+2 \mathrm{~b}+\mathrm{d})
\end{aligned}
$$

Formula (1) is equal to the formula proposed by Delgado, Vila, and Voxman et al. [8].

The triangular fuzzy number $\mathrm{Y}=(\mathrm{c}, \mathrm{a}, \mathrm{b})$ is a special case of generalized trapezoidal fuzzy number. The graded mean integration representation of triangular fuzzy number $\mathrm{Y}$ becomes

$$
P(Y)=\frac{1}{6}(c+4 a+b)
$$




\subsection{The Inverse Function Arithmetic Representation Method}

Based on the above graded mean integration representation method, we further propose the Inverse Function Arithmetic Presentation Method for the multiplication operation on multiple fuzzy numbers as follows

Suppose the membership function of $A_{1}=\left(c_{1}, a_{1}, b_{1}\right)$ is

$$
f_{A 1}(x)= \begin{cases}\frac{\left(x-c_{1}\right)}{\left(a_{1}-c_{1}\right)}, & c_{1} \leq x \leq a_{1}, \\ \frac{\left(x-b_{1}\right)}{\left(a_{1}-b_{1}\right)}, & a_{1} \leq x \leq b_{1}, \\ 0, \text { otherwise } & \end{cases}
$$

Since $\quad L_{A 1}(x)=\frac{\left(x-c_{1}\right)}{\left(a_{1}-c_{1}\right)}, \quad c_{1} \leq x \leq a_{1}, \quad R_{A 1}(x)=\frac{\left(x-b_{1}\right)}{\left(a_{1}-b_{1}\right)}, \quad a_{1} \leq x \leq b_{1}$,

and $\quad \mathrm{L}^{-1}{ }_{\mathrm{Al}}(\mathrm{h})=\mathrm{c}_{1}+\left(\mathrm{a}_{1}-\mathrm{c}_{1}\right) \mathrm{h}, \quad 0 \leq \mathrm{h} \leq 1, \quad \mathrm{R}^{-1}{ }_{\mathrm{Al}}(\mathrm{h})=\mathrm{b}_{1}+\left(\mathrm{a}_{1}-\mathrm{b}_{1}\right) \mathrm{h}, \quad 0 \leq \mathrm{h} \leq 1$

$\mathrm{L}_{\mathrm{Al}}(\mathrm{x})$ and $\mathrm{R}_{\mathrm{A} 1}(\mathrm{x})$ are the function $\mathrm{L}$ and function $\mathrm{R}$ of fuzzy number $\mathrm{A}_{1}$ respectively. $\mathrm{L}^{-1}{ }_{\mathrm{Al}}(\mathrm{h})$ and $\mathrm{R}^{-1}{ }_{\mathrm{Al}}(\mathrm{h})$ are the inverse functions of function $\mathrm{L}_{\mathrm{Al}}(\mathrm{x})$ and function $\mathrm{R}_{\mathrm{Al}}(\mathrm{x})$ at h-level, respectively.

Similarly, suppose the membership function of $A_{2}=\left(c_{2}, a_{2}, b_{2}\right)$ is

$$
f_{A 2}(x)= \begin{cases}\frac{\left(x-c_{2}\right)}{\left(a_{2}-c_{2}\right)}, & c_{2} \leq x \leq a_{2}, \\ \frac{\left(x-b_{2}\right)}{\left(a_{2}-b_{2}\right)}, & a_{2} \leq x \leq b_{2}, \\ 0, \text { otherwise } & \end{cases}
$$

Since $\quad \mathrm{L}_{\mathrm{A} 2}(\mathrm{x})=\frac{\left(\mathrm{x}-\mathrm{c}_{2}\right)}{\left(\mathrm{a}_{2}-\mathrm{c}_{2}\right)}, \quad \mathrm{c}_{2} \leq \mathrm{x} \leq \mathrm{a}_{2}, \quad \mathrm{R}_{\mathrm{A} 2}(\mathrm{x})=\frac{\left(\mathrm{x}-\mathrm{b}_{2}\right)}{\left(\mathrm{a}_{2}-\mathrm{b}_{2}\right)}, \quad \mathrm{a}_{2} \leq \mathrm{x} \leq \mathrm{b}_{2}$,

and $\quad \mathrm{L}^{-1}{ }_{\mathrm{A} 2}(\mathrm{~h})=\mathrm{c}_{2}+\left(\mathrm{a}_{2}-\mathrm{c}_{2}\right) \mathrm{h}, \quad 0 \leq \mathrm{h} \leq 1, \quad \mathrm{R}^{-1}{ }_{\mathrm{A} 2}(\mathrm{~h})=\mathrm{b}_{2}+\left(\mathrm{a}_{2}-\mathrm{b}_{2}\right) \mathrm{h}, \quad 0 \leq \mathrm{h} \leq 1$.

$\mathrm{L}_{\mathrm{A} 2}(\mathrm{x})$ and $\mathrm{R}_{\mathrm{A} 2}(\mathrm{x})$ are the function $\mathrm{L}$ and function $\mathrm{R}$ of fuzzy number $\mathrm{A}_{2}$ respectively. $\mathrm{L}^{-1}{ }_{\mathrm{A} 2}(\mathrm{~h})$ and $\mathrm{R}^{-1}{ }_{\mathrm{A} 2}(\mathrm{~h})$ are the inverse functions of function $\mathrm{L}_{\mathrm{A} 2}(\mathrm{x})$ and $\mathrm{R}_{\mathrm{A} 2}(\mathrm{x})$ at h-level. Similarly, suppose the membership function of $A_{3}=\left(c_{3}, a_{3}, b_{3}\right)$ is

$$
f_{A 3}(x)= \begin{cases}\frac{\left(x-c_{3}\right)}{\left(a_{3}-c_{3}\right)}, & c_{3} \leq x \leq a_{3}, \\ \frac{\left(x-b_{3}\right)}{\left(a_{3}-b_{3}\right)}, & a_{3} \leq x \leq b_{3}, \\ 0, \text { otherwise } & \text {. }\end{cases}
$$

Since $\quad L_{A 3}(x)=\frac{\left(x-c_{3}\right)}{\left(a_{3}-c_{3}\right)}, \quad c_{3} \leq x \leq a_{3}, \quad R_{A 3}(x)=\frac{\left(x-b_{3}\right)}{\left(a_{3}-b_{3}\right)}, \quad a_{3} \leq x \leq b_{3}$, and $\quad \mathrm{L}^{-1}{ }_{\mathrm{A} 3}(\mathrm{~h})=\mathrm{c}_{3}+\left(\mathrm{a}_{3}-\mathrm{c}_{3}\right) \mathrm{h}, \quad 0 \leq \mathrm{h} \leq 1, \quad \mathrm{R}^{-1}{ }_{\mathrm{A} 3}(\mathrm{~h})=\mathrm{b}_{3}+\left(\mathrm{a}_{3}-\mathrm{b}_{3}\right) \mathrm{h}, \quad 0 \leq \mathrm{h} \leq 1$.

$\mathrm{L}_{\mathrm{A} 3}(\mathrm{x})$ and $\mathrm{R}_{\mathrm{A} 3}(\mathrm{x})$ are the function $\mathrm{L}$ and function $\mathrm{R}$ of fuzzy number $\mathrm{A}_{3}$ respectively. $\mathrm{L}^{-1}{ }_{\mathrm{A} 3}(\mathrm{~h})$ and $\mathrm{R}^{-1}{ }_{\mathrm{A} 3}(\mathrm{~h})$ are the inverse functions of function $\mathrm{L}_{\mathrm{A} 3}(\mathrm{x})$ and $\mathrm{R}_{\mathrm{A} 3}(\mathrm{x})$ at h-level. 
Definition 1. Let $\mathrm{A}_{1}=\left(\mathrm{c}_{1}, \mathrm{a}_{1}, \mathrm{~b}_{1}\right), \mathrm{A}_{2}=\left(\mathrm{c}_{2}, \mathrm{a}_{2}, \mathrm{~b}_{2}\right)$ and $\mathrm{A}_{3}=\left(\mathrm{c}_{3}, \mathrm{a}_{3}, \mathrm{~b}_{3}\right)$ be all triangular fuzzy numbers. The representation of multiplication operation on multiple triangular fuzzy numbers at h-level is

$$
\begin{aligned}
& \mathrm{A}_{1(\mathrm{~h})} \otimes \mathrm{A}_{2(\mathrm{~h})} \otimes \mathrm{A}_{3(\mathrm{~h})}=\frac{1}{8} \mathrm{~h} \times\left[\mathrm{L}_{\mathrm{Al}}^{-1}(\mathrm{~h}) \times \mathrm{L}_{\mathrm{A} 2}^{-1}(\mathrm{~h}) \times \mathrm{L}_{\mathrm{A} 3}^{-1}(\mathrm{~h})+\mathrm{R}^{-1}{ }_{\mathrm{A} 1}(\mathrm{~h}) \times \mathrm{L}_{\mathrm{A} 2}^{-1}(\mathrm{~h}) \times \mathrm{L}^{-1}{ }_{\mathrm{A} 3}(\mathrm{~h})\right. \\
& +\mathrm{L}^{-1}{ }_{\mathrm{A} 1}(\mathrm{~h}) \times \mathrm{R}^{-1}{ }_{\mathrm{A} 2}(\mathrm{~h}) \times \mathrm{L}^{-1}{ }_{\mathrm{A} 3}(\mathrm{~h})+\mathrm{L}^{-1} \mathrm{Al}_{1}(\mathrm{~h}) \times \mathrm{L}^{-1}{ }_{\mathrm{A} 2}(\mathrm{~h}) \times \mathrm{R}^{-1}{ }_{\mathrm{A} 3}(\mathrm{~h}) \\
& +\mathrm{L}^{-1}{ }_{\mathrm{A} 1}(\mathrm{~h}) \times \mathrm{R}^{-1}{ }_{\mathrm{A} 2}(\mathrm{~h}) \times \mathrm{R}^{-1}{ }_{\mathrm{A} 3}(\mathrm{~h})+\mathrm{R}^{-1}{ }_{\mathrm{A} 1}(\mathrm{~h}) \times \mathrm{L}^{-1}{ }_{\mathrm{A} 2}(\mathrm{~h}) \times \mathrm{R}^{-1}{ }_{\mathrm{A} 3}(\mathrm{~h}) \\
& \left.+\mathrm{R}^{-1}{ }_{\mathrm{A} 1}(\mathrm{~h}) \times \mathrm{R}_{\mathrm{A} 2}^{-1}(\mathrm{~h}) \times \mathrm{L}_{\mathrm{A} 3}^{-1}(\mathrm{~h})+\mathrm{R}_{\mathrm{A} 1}^{-1}(\mathrm{~h}) \times \mathrm{R}_{\mathrm{A} 2}^{-1}(\mathrm{~h}) \times \mathrm{R}^{-1}{ }_{\mathrm{A} 3}(\mathrm{~h})\right]
\end{aligned}
$$

Definition 2. Let $\mathrm{P}\left(\mathrm{A}_{1} \otimes \mathrm{A}_{2} \otimes \mathrm{A}_{3}\right)$ be the representation of $\mathrm{A}_{1} \otimes \mathrm{A}_{2} \otimes \mathrm{A}_{3}$. And $\mathrm{P}\left(\mathrm{A}_{1} \otimes \mathrm{A}_{2} \otimes \mathrm{A}_{3}\right)$ is defined as follows.

$$
\begin{aligned}
& \mathrm{P}\left(\mathrm{A}_{1} \otimes \mathrm{A}_{2} \otimes \mathrm{A}_{3}\right) \\
& =\int_{0}^{1} \int_{0}^{1} \int_{0}^{1} \frac{1}{8}\left[\left(\mathrm{~h}_{\mathrm{A} 1} \mathrm{~L}_{\mathrm{A} 1}^{-1}(\mathrm{~h}) \times \mathrm{h}_{\mathrm{A} 2} \mathrm{~L}_{\mathrm{A} 2}^{-1}(\mathrm{~h}) \times \mathrm{h}_{\mathrm{A} 3} \mathrm{~L}_{\mathrm{A} 3}^{-1}(\mathrm{~h})\right)+\left(\mathrm{h}_{\mathrm{A} 1} \mathrm{R}_{\mathrm{Al}}^{-1}(\mathrm{~h}) \times \mathrm{h}_{\mathrm{A} 2} \mathrm{~L}^{-1}{ }_{\mathrm{A} 2}(\mathrm{~h}) \times \mathrm{h}_{\mathrm{A} 3} \mathrm{~L}_{\mathrm{A} 3}^{-1}(\mathrm{~h})\right)\right. \\
& +\left(\mathrm{h}_{\mathrm{A} 1} \mathrm{~L}_{\mathrm{A} 1}^{-1}(\mathrm{~h}) \times \mathrm{h}_{\mathrm{A} 2} \mathrm{R}^{-1}{ }_{\mathrm{A} 2}(\mathrm{~h}) \times \mathrm{h}_{\mathrm{A} 3} \mathrm{~L}^{-1}{ }_{\mathrm{A} 3}(\mathrm{~h})\right)+\left(\mathrm{h}_{\mathrm{A} 1} \mathrm{~L}^{-1}{ }_{\mathrm{A} 1}(\mathrm{~h}) \times \mathrm{h}_{\mathrm{A} 2} \mathrm{~L}^{-1}{ }_{\mathrm{A} 2}(\mathrm{~h}) \times \mathrm{h}_{\mathrm{A} 3} \mathrm{R}^{-1}{ }_{\mathrm{A} 3}(\mathrm{~h})\right) \\
& +\left(\mathrm{h}_{\mathrm{A} 1} \mathrm{~L}^{-1}{ }_{\mathrm{A} 1}(\mathrm{~h}) \times \mathrm{h}_{\mathrm{A} 2} \mathrm{R}^{-1}{ }_{\mathrm{A} 2}(\mathrm{~h}) \times \mathrm{h}_{\mathrm{A} 3} \mathrm{R}^{-1}{ }_{\mathrm{A} 3}(\mathrm{~h})\right)+\left(\mathrm{h}_{\mathrm{A} 1} \mathrm{R}^{-1}{ }_{\mathrm{A} 1}(\mathrm{~h}) \times \mathrm{h}_{\mathrm{A} 2} \mathrm{~L}^{-1}{ }_{\mathrm{A} 2}(\mathrm{~h}) \times \mathrm{h}_{\mathrm{A} 3} \mathrm{R}_{\mathrm{A} 3}(\mathrm{~h})\right) \\
& \left.+\left(\mathrm{h}_{\mathrm{A} 1} \mathrm{R}^{-1}{ }_{\mathrm{A} 1}(\mathrm{~h}) \times \mathrm{h}_{\mathrm{A} 2} \mathrm{R}^{-1}{ }_{\mathrm{A} 2}(\mathrm{~h}) \times \mathrm{h}_{\mathrm{A} 3} \mathrm{~L}^{-1}{ }_{\mathrm{A} 3}(\mathrm{~h})\right)+\left(\mathrm{h}_{\mathrm{A} 1} \mathrm{R}^{-1}{ }_{\mathrm{A} 1}(\mathrm{~h}) \times \mathrm{h}_{\mathrm{A} 2} \mathrm{R}^{-1}{ }_{\mathrm{A} 2}(\mathrm{~h}) \times \mathrm{h}_{\mathrm{A} 3} \mathrm{R}^{-1}{ }_{\mathrm{A} 3}(\mathrm{~h})\right)\right] \\
& \mathrm{dh}_{\mathrm{A} 1} \mathrm{dh}_{\mathrm{A} 2} \mathrm{dh}_{\mathrm{A} 3} /\left(\int_{0}^{1} \mathrm{~h}_{\mathrm{A} 1} \mathrm{dh}_{\mathrm{A} 1} \times \int_{0}^{1} \mathrm{~h}_{\mathrm{A} 2} \mathrm{dh}_{\mathrm{A} 2} \times \int_{0}^{1} \mathrm{~h}_{\mathrm{A} 3} \mathrm{dh}_{\mathrm{A} 3}\right)
\end{aligned}
$$

By formula (3),

$\mathrm{P}\left(\mathrm{A}_{1} \otimes \mathrm{A}_{2} \otimes \mathrm{A}_{3}\right)$

$=\int_{0}^{1} \int_{0}^{1} \int_{0}^{1} \frac{1}{8}\left[\left(\mathrm{~h}_{\mathrm{Al}} \mathrm{L}^{-1}{ }_{\mathrm{Al}}(\mathrm{h}) \times \mathrm{h}_{\mathrm{A} 2} \mathrm{~L}^{-1}{ }_{\mathrm{A} 2}(\mathrm{~h}) \times \mathrm{h}_{\mathrm{A} 3} \mathrm{~L}^{-1}{ }_{\mathrm{A} 3}(\mathrm{~h})\right)+\left(\mathrm{h}_{\mathrm{A} 1} \mathrm{R}^{-1}{ }_{\mathrm{Al}}(\mathrm{h}) \times \mathrm{h}_{\mathrm{A} 2} \mathrm{~L}^{-1}{ }_{\mathrm{A} 2}(\mathrm{~h}) \times \mathrm{h}_{\mathrm{A} 3} \mathrm{~L}^{-1}{ }_{\mathrm{A} 3}(\mathrm{~h})\right)\right.$

$+\left(\mathrm{h}_{\mathrm{A} 1} \mathrm{~L}^{-1}{ }_{\mathrm{A} 1}(\mathrm{~h}) \times \mathrm{h}_{\mathrm{A} 2} \mathrm{R}^{-1}{ }_{\mathrm{A} 2}(\mathrm{~h}) \times \mathrm{h}_{\mathrm{A} 3} \mathrm{~L}^{-1}{ }_{\mathrm{A} 3}(\mathrm{~h})\right)+\left(\mathrm{h}_{\mathrm{A} 1} \mathrm{~L}^{-1}{ }_{\mathrm{A} 1}(\mathrm{~h}) \times \mathrm{h}_{\mathrm{A} 2} \mathrm{~L}^{-1}{ }_{\mathrm{A} 2}(\mathrm{~h}) \times \mathrm{h}_{\mathrm{A} 3} \mathrm{R}^{-1}{ }_{\mathrm{A} 3}(\mathrm{~h})\right)$

$+\left(h_{\mathrm{A} 1} \mathrm{~L}^{-1}{ }_{\mathrm{A} 1}(\mathrm{~h}) \times \mathrm{h}_{\mathrm{A} 2} \mathrm{R}^{-1}{ }_{\mathrm{A} 2}(\mathrm{~h}) \times \mathrm{h}_{\mathrm{A} 3} \mathrm{R}^{-1}{ }_{\mathrm{A} 3}(\mathrm{~h})\right)+\left(\mathrm{h}_{\mathrm{A} 1} \mathrm{R}^{-1}{ }_{\mathrm{A} 1}(\mathrm{~h}) \times \mathrm{h}_{\mathrm{A} 2} \mathrm{~L}^{-1}{ }_{\mathrm{A} 2}(\mathrm{~h}) \times \mathrm{h}_{\mathrm{A} 3} \mathrm{R}^{-1}{ }_{\mathrm{A} 3}(\mathrm{~h})\right)$

$\left.+\left(\mathrm{h}_{\mathrm{A} 1} \mathrm{R}^{-1}{ }_{\mathrm{A} 1}(\mathrm{~h}) \times \mathrm{h}_{\mathrm{A} 2} \mathrm{R}^{-1}{ }_{\mathrm{A} 2}(\mathrm{~h}) \times \mathrm{h}_{\mathrm{A} 3} \mathrm{~L}^{-1}{ }_{\mathrm{A} 3}(\mathrm{~h})\right)+\left(\mathrm{h}_{\mathrm{A} 1} \mathrm{R}^{-1}{ }_{\mathrm{A} 1}(\mathrm{~h}) \times \mathrm{h}_{\mathrm{A} 2} \mathrm{R}^{-1}{ }_{\mathrm{A} 2}(\mathrm{~h}) \times \mathrm{h}_{\mathrm{A} 3} \mathrm{R}^{-1}{ }_{\mathrm{A} 3}(\mathrm{~h})\right)\right]$

$\mathrm{dh}_{\mathrm{A} 1} \mathrm{dh}_{\mathrm{A} 2} \mathrm{dh}_{\mathrm{A} 3} /\left(\int_{0}^{1} \mathrm{~h}_{\mathrm{A} 1} \mathrm{dh}_{\mathrm{A} 1} \times \int_{0}^{1} \mathrm{~h}_{\mathrm{A} 2} \mathrm{dh}_{\mathrm{A} 2} \times \int_{0}^{1} \mathrm{~h}_{\mathrm{A} 3} \mathrm{dh}_{\mathrm{A} 3}\right)$

$=\int_{0}^{1} \int_{0}^{1} \int_{0}^{1} \frac{1}{8}\left\{\mathrm{~h}_{\mathrm{A} 1}\left[\mathrm{c}_{1}+\left(\mathrm{a}_{1}-\mathrm{c}_{1}\right) \mathrm{h}_{\mathrm{A} 1}\right] \times \mathrm{h}_{\mathrm{A} 2}\left[\mathrm{c}_{2}+\left(\mathrm{a}_{2}-\mathrm{c}_{2}\right) \mathrm{h}_{\mathrm{A} 2}\right] \times \mathrm{h}_{\mathrm{A} 3}\left[\mathrm{c}_{3}+\left(\mathrm{a}_{3}-\mathrm{c}_{3}\right) \mathrm{h}_{\mathrm{A} 3}\right]\right.$

$+\mathrm{h}_{\mathrm{A} 1}\left[\mathrm{~b}_{1}+\left(\mathrm{a}_{1}-\mathrm{b}_{1}\right) \mathrm{h}_{\mathrm{A} 1}\right] \times \mathrm{h}_{\mathrm{A} 2}\left[\mathrm{c}_{2}+\left(\mathrm{a}_{2}-\mathrm{c}_{2}\right) \mathrm{h}_{\mathrm{A} 2}\right] \times \mathrm{h}_{\mathrm{A} 3}\left[\mathrm{c}_{3}+\left(\mathrm{a}_{3}-\mathrm{c}_{3}\right) \mathrm{h}_{\mathrm{A} 3}\right]$

$+\mathrm{h}_{\mathrm{A} 1}\left[\mathrm{c}_{1}+\left(\mathrm{a}_{1}-\mathrm{c}_{1}\right) \mathrm{h}_{\mathrm{A} 1}\right] \times \mathrm{h}_{\mathrm{A} 2}\left[\mathrm{~b}_{2}+\left(\mathrm{a}_{2}-\mathrm{b}_{2}\right) \mathrm{h}_{\mathrm{A} 2}\right] \times \mathrm{h}_{\mathrm{A} 3}\left[\mathrm{c}_{3}+\left(\mathrm{a}_{3}-\mathrm{c}_{3}\right) \mathrm{h}_{\mathrm{A} 3}\right]$

$+\mathrm{h}_{\mathrm{A} 1}\left[\mathrm{c}_{1}+\left(\mathrm{a}_{1}-\mathrm{c}_{1}\right) \mathrm{h}_{\mathrm{A} 1}\right] \times \mathrm{h}_{\mathrm{A} 2}\left[\mathrm{c}_{2}+\left(\mathrm{a}_{2}-\mathrm{c}_{2}\right) \mathrm{h}_{\mathrm{A} 2}\right] \times \mathrm{h}_{\mathrm{A} 3}\left[\mathrm{~b}_{3}+\left(\mathrm{a}_{3}-\mathrm{b}_{3}\right) \mathrm{h}_{\mathrm{A} 3}\right]$

$+\mathrm{h}_{\mathrm{A} 1}\left[\mathrm{c}_{1}+\left(\mathrm{a}_{1}-\mathrm{c}_{1}\right) \mathrm{h}_{\mathrm{A} 1}\right] \times \mathrm{h}_{\mathrm{A} 2}\left[\mathrm{~b}_{2}+\left(\mathrm{a}_{2}-\mathrm{b}_{2}\right) \mathrm{h}_{\mathrm{A} 2}\right] \times \mathrm{h}_{\mathrm{A} 3}\left[\mathrm{~b}_{3}+\left(\mathrm{a}_{3}-\mathrm{b}_{3}\right) \mathrm{h}_{\mathrm{A} 3}\right]$

$+\mathrm{h}_{\mathrm{A} 1}\left[\mathrm{~b}_{1}+\left(\mathrm{a}_{1}-\mathrm{b}_{1}\right) \mathrm{h}_{\mathrm{A} 1}\right] \times \mathrm{h}_{\mathrm{A} 2}\left[\mathrm{c}_{2}+\left(\mathrm{a}_{2}-\mathrm{c}_{2}\right) \mathrm{h}_{\mathrm{A} 2}\right] \times \mathrm{h}_{\mathrm{A} 3}\left[\mathrm{~b}_{3}+\left(\mathrm{a}_{3}-\mathrm{b}_{3}\right) \mathrm{h}_{\mathrm{A} 3}\right]$

$+\mathrm{h}_{\mathrm{A} 1}\left[\mathrm{~b}_{1}+\left(\mathrm{a}_{1}-\mathrm{b}_{1}\right) \mathrm{h}_{\mathrm{A} 1}\right] \times \mathrm{h}_{\mathrm{A} 2}\left[\mathrm{~b}_{2}+\left(\mathrm{a}_{2}-\mathrm{b}_{2}\right) \mathrm{h}_{\mathrm{A} 2}\right] \times \mathrm{h}_{\mathrm{A} 3}\left[\mathrm{c}_{3}+\left(\mathrm{a}_{3}-\mathrm{c}_{3}\right) \mathrm{h}_{\mathrm{A} 3}\right]$

$\left.+\mathrm{h}_{\mathrm{A} 1}\left[\mathrm{~b}_{1}+\left(\mathrm{a}_{1}-\mathrm{b}_{1}\right) \mathrm{h}_{\mathrm{A} 1}\right] \times \mathrm{h}_{\mathrm{A} 2}\left[\mathrm{~b}_{2}+\left(\mathrm{a}_{2}-\mathrm{b}_{2}\right) \mathrm{h}_{\mathrm{A} 2}\right] \times \mathrm{h}_{\mathrm{A} 3}\left[\mathrm{~b}_{3}+\left(\mathrm{a}_{3}-\mathrm{b}_{3}\right) \mathrm{h}_{\mathrm{A} 3}\right]\right\}$

$\mathrm{dh}_{\mathrm{A} 1} \mathrm{dh}_{\mathrm{A} 2} \mathrm{dh}_{\mathrm{A} 3} /\left(\int_{0}^{1} \mathrm{~h}_{\mathrm{A} 1} \mathrm{dh}_{\mathrm{A} 1} \times \int_{0}^{1} \mathrm{~h}_{\mathrm{A} 2} \mathrm{dh}_{\mathrm{A} 2} \times \int_{0}^{1} \mathrm{~h}_{\mathrm{A} 3} \mathrm{dh}_{\mathrm{A} 3}\right)$

$=\int_{0}^{1} \int_{0}^{1} \frac{1}{8}\left\{\left[\frac{1}{2} \mathrm{c}_{1} \mathrm{~h}_{\mathrm{A} 1}^{2}+\frac{1}{3}\left(\mathrm{a}_{1}-\mathrm{c}_{1}\right) \mathrm{h}_{\mathrm{A} 1}^{3}\right] \times \mathrm{h}_{\mathrm{A} 2}\left[\mathrm{c}_{2}+\left(\mathrm{a}_{2}-\mathrm{c}_{2}\right) \mathrm{h}_{\mathrm{A} 2}\right] \times \mathrm{h}_{\mathrm{A} 3}\left[\mathrm{c}_{3}+\left(\mathrm{a}_{3}-\mathrm{c}_{3}\right) \mathrm{h}_{\mathrm{A} 3}\right]\right.$ 
$+\left[\frac{1}{2} \mathrm{~b}_{1} \mathrm{~h}_{\mathrm{A1}}{ }^{2}+\frac{1}{3}\left(\mathrm{a}_{1}-\mathrm{b}_{1}\right) \mathrm{h}_{\mathrm{Al}}{ }^{3}\right] \times \mathrm{h}_{\mathrm{A} 2}\left[\mathrm{c}_{2}+\left(\mathrm{a}_{2}-\mathrm{c}_{2}\right) \mathrm{h}_{\mathrm{A} 2}\right] \times \mathrm{h}_{\mathrm{A} 3}\left[\mathrm{c}_{3}+\left(\mathrm{a}_{3}-\mathrm{c}_{3}\right) \mathrm{h}_{\mathrm{A} 3}\right]$ $+\left[\frac{1}{2} \mathrm{c}_{1} \mathrm{~h}_{\mathrm{A1}}{ }^{2}+\frac{1}{3}\left(\mathrm{a}_{1}-\mathrm{c}_{1}\right) \mathrm{h}_{\mathrm{A1}}{ }^{3}\right] \times \mathrm{h}_{\mathrm{A} 2}\left[\mathrm{~b}_{2}+\left(\mathrm{a}_{2}-\mathrm{b}_{2}\right) \mathrm{h}_{\mathrm{A} 2}\right] \times \mathrm{h}_{\mathrm{A} 3}\left[\mathrm{c}_{3}+\left(\mathrm{a}_{3}-\mathrm{c}_{3}\right) \mathrm{h}_{\mathrm{A} 3}\right]$ $+\left[\frac{1}{2} c_{1} h_{A 1}{ }^{2}+\frac{1}{3}\left(a_{1}-c_{1}\right) h_{A 1}{ }^{3}\right] \times h_{A^{2}}\left[c_{2}+\left(a_{2}-c_{2}\right) h_{A 2}\right] \times h_{A 3}\left[b_{3}+\left(a_{3}-b_{3}\right) h_{A 3}\right]$ $+\left[\frac{1}{2} c_{1} h_{A 1}{ }^{2}+\frac{1}{3}\left(a_{1}-c_{1}\right) h_{A 1}{ }^{3}\right] \times h_{A 2}\left[b_{2}+\left(a_{2}-b_{2}\right) h_{A 2}\right] \times h_{A 3}\left[b_{3}+\left(a_{3}-b_{3}\right) h_{A 3}\right]$ $+\left[\frac{1}{2} \mathrm{~b}_{1} \mathrm{~h}_{\mathrm{Al}}{ }^{2}+\frac{1}{3}\left(\mathrm{a}_{1}-\mathrm{b}_{1}\right) \mathrm{h}_{\mathrm{Al}}{ }^{3}\right] \times \mathrm{h}_{\mathrm{A} 2}\left[\mathrm{c}_{2}+\left(\mathrm{a}_{2}-\mathrm{c}_{2}\right) \mathrm{h}_{\mathrm{A} 2}\right] \times \mathrm{h}_{\mathrm{A3}}\left[\mathrm{b}_{3}+\left(\mathrm{a}_{3}-\mathrm{b}_{3}\right) \mathrm{h}_{\mathrm{A} 3}\right]$ $+\left[\frac{1}{2} \mathrm{~b}_{1} \mathrm{~h}_{\mathrm{Al}}{ }^{2}+\frac{1}{3}\left(\mathrm{a}_{1}-\mathrm{b}_{1}\right) \mathrm{h}_{\mathrm{A1}}{ }^{3}\right] \times \mathrm{h}_{\mathrm{A} 2}\left[\mathrm{~b}_{2}+\left(\mathrm{a}_{2}-\mathrm{b}_{2}\right) \mathrm{h}_{\mathrm{A} 2}\right] \times \mathrm{h}_{\mathrm{A} 3}\left[\mathrm{c}_{3}+\left(\mathrm{a}_{3}-\mathrm{c}_{3}\right) \mathrm{h}_{\mathrm{A} 3}\right]$ $\left.+\left[\frac{1}{2} b_{1} h_{A 1}{ }^{2}+\frac{1}{3}\left(a_{1}-b_{1}\right) h_{A 1}^{3}\right] \times h_{A 2}\left[b_{2}+\left(a_{2}-b_{2}\right) h_{A 2}\right] \times h_{A 3}\left[b_{3}+\left(a_{3}-b_{3}\right) h_{A 3}\right]\right\}||_{0}^{1} d_{\mathrm{A}_{2}} \mathrm{dh}_{\mathrm{A} 3}$ $/\left(\frac{1}{2} \mathrm{~h}_{\mathrm{A} 1}^{2}||_{0}^{1} \times \int_{0}^{1} \mathrm{~h}_{\mathrm{A} 2} \mathrm{dh}_{\mathrm{A} 2} \times \int_{0}^{1} \mathrm{~h}_{\mathrm{A} 3} \mathrm{dh}_{\mathrm{A} 3}\right)$ $=\int_{0}^{1} \int_{0}^{1} \frac{1}{8}\left\{\left[\frac{1}{2} \mathrm{c}_{1}+\frac{1}{3}\left(\mathrm{a}_{1}-\mathrm{c}_{1}\right)\right] \times \mathrm{h}_{\mathrm{A} 2}\left[\mathrm{c}_{2}+\left(\mathrm{a}_{2}-\mathrm{c}_{2}\right) \mathrm{h}_{\mathrm{A} 2}\right] \times \mathrm{h}_{\mathrm{A} 3}\left[\mathrm{c}_{3}+\left(\mathrm{a}_{3}-\mathrm{c}_{3}\right) \mathrm{h}_{\mathrm{A} 3}\right]\right.$ $+\left[\frac{1}{2} \mathrm{~b}_{1}+\frac{1}{3}\left(\mathrm{a}_{1}-\mathrm{b}_{1}\right)\right] \times \mathrm{h}_{\mathrm{A} 2}\left[\mathrm{c}_{2}+\left(\mathrm{a}_{2}-\mathrm{c}_{2}\right) \mathrm{h}_{\mathrm{A} 2}\right] \times \mathrm{h}_{\mathrm{A} 3}\left[\mathrm{c}_{3}+\left(\mathrm{a}_{3}-\mathrm{c}_{3}\right) \mathrm{h}_{\mathrm{A} 3}\right]$ $+\left[\frac{1}{2} c_{1}+\frac{1}{3}\left(a_{1}-c_{1}\right)\right] \times h_{A 2}\left[b_{2}+\left(a_{2}-b_{2}\right) h_{A 2}\right] \times h_{A 3}\left[c_{3}+\left(a_{3}-c_{3}\right) h_{A 3}\right]$ $+\left[\frac{1}{2} c_{1}+\frac{1}{3}\left(a_{1}-c_{1}\right)\right] \times h_{A 2}\left[c_{2}+\left(a_{2}-c_{2}\right) h_{A 2}\right] \times h_{A 3}\left[b_{3}+\left(a_{3}-b_{3}\right) h_{A 3}\right]$ $+\left[\frac{1}{2} c_{1}+\frac{1}{3}\left(a_{1}-c_{1}\right)\right] \times h_{A 2}\left[b_{2}+\left(a_{2}-b_{2}\right) h_{A 2}\right] \times h_{A 3}\left[b_{3}+\left(a_{3}-b_{3}\right) h_{A 3}\right]$ $+\left[\frac{1}{2} b_{1}+\frac{1}{3}\left(a_{1}-b_{1}\right)\right] \times h_{A_{2} 2}\left[c_{2}+\left(a_{2}-c_{2}\right) h_{A 2}\right] \times h_{A 3}\left[b_{3}+\left(a_{3}-b_{3}\right) h_{A 3}\right]$ $+\left[\frac{1}{2} \mathrm{~b}_{1}+\frac{1}{3}\left(\mathrm{a}_{1}-\mathrm{b}_{1}\right)\right] \times \mathrm{h}_{\mathrm{A} 2}\left[\mathrm{~b}_{2}+\left(\mathrm{a}_{2}-\mathrm{b}_{2}\right) \mathrm{h}_{\mathrm{A} 2}\right] \times \mathrm{h}_{\mathrm{A} 3}\left[\mathrm{c}_{3}+\left(\mathrm{a}_{3}-\mathrm{c}_{3}\right) \mathrm{h}_{\mathrm{A} 3}\right]$ $\left.+\left[\frac{1}{2} b_{1}+\frac{1}{3}\left(a_{1}-b_{1}\right)\right] \times h_{\mathrm{A} 2}\left[b_{2}+\left(a_{2}-b_{2}\right) h_{\mathrm{A} 2}\right] \times h_{\mathrm{A} 3}\left[b_{3}+\left(a_{3}-b_{3}\right) h_{\mathrm{A} 3}\right]\right\} \mathrm{dh}_{\mathrm{A} 2} \mathrm{dh}_{\mathrm{A} 3}$ $/\left(\frac{1}{2} \times \int_{0}^{1} \mathrm{~h}_{\mathrm{A} 2} \mathrm{dh}_{\mathrm{A} 2} \times \int_{0}^{1} \mathrm{~h}_{\mathrm{A} 3} \mathrm{dh}_{\mathrm{A} 3}\right)$ $=\int_{0}^{1} \frac{1}{8}\left\{\left[\frac{1}{2} c_{1}+\frac{1}{3}\left(a_{1}-c_{1}\right)\right] \times\left[\frac{1}{2} c_{2}+\frac{1}{3}\left(a_{2}-c_{2}\right)\right] \times \mathrm{h}_{A 3}\left[c_{3}+\left(a_{3}-c_{3}\right) h_{A 3}\right]\right.$ $+\left[\frac{1}{2} b_{1}+\frac{1}{3}\left(a_{1}-b_{1}\right)\right] \times\left[\frac{1}{2} c_{2}+\frac{1}{3}\left(a_{2}-c_{2}\right)\right] \times h_{A 3}\left[c_{3}+\left(a_{3}-c_{3}\right) h_{A 3}\right]$ $+\left[\frac{1}{2} c_{1}+\frac{1}{3}\left(a_{1}-c_{1}\right)\right] \times\left[\frac{1}{2} b_{2}+\frac{1}{3}\left(a_{2}-b_{2}\right)\right] \times h_{A 3}\left[c_{3}+\left(a_{3}-c_{3}\right) h_{A 3}\right]$ $+\left[\frac{1}{2} c_{1}+\frac{1}{3}\left(a_{1}-c_{1}\right)\right] \times\left[\frac{1}{2} c_{2}+\frac{1}{3}\left(a_{2}-c_{2}\right)\right] \times h_{A 3}\left[b_{3}+\left(a_{3}-b_{3}\right) h_{A 3}\right]$ 
$+\left[\frac{1}{2} c_{1}+\frac{1}{3}\left(a_{1}-c_{1}\right)\right] \times\left[\frac{1}{2} b_{2}+\frac{1}{3}\left(a_{2}-b_{2}\right)\right] \times h_{A 3}\left[b_{3}+\left(a_{3}-b_{3}\right) h_{\mathrm{A} 3}\right]$

$+\left[\frac{1}{2} \mathrm{~b}_{1}+\frac{1}{3}\left(\mathrm{a}_{1}-\mathrm{b}_{1}\right)\right] \times\left[\frac{1}{2} \mathrm{c}_{2}+\frac{1}{3}\left(\mathrm{a}_{2}-\mathrm{c}_{2}\right)\right] \times \mathrm{h}_{\mathrm{A} 3}\left[\mathrm{~b}_{3}+\left(\mathrm{a}_{3}-\mathrm{b}_{3}\right) \mathrm{h}_{\mathrm{A} 3}\right]$

$+\left[\frac{1}{2} \mathrm{~b}_{1}+\frac{1}{3}\left(\mathrm{a}_{1}-\mathrm{b}_{1}\right)\right] \times\left[\frac{1}{2} \mathrm{~b}_{2}+\frac{1}{3}\left(\mathrm{a}_{2}-\mathrm{b}_{2}\right)\right] \times \mathrm{h}_{\mathrm{A} 3}\left[\mathrm{c}_{3}+\left(\mathrm{a}_{3}-\mathrm{c}_{3}\right) \mathrm{h}_{\mathrm{A} 3}\right]$

$\left.+\left[\frac{1}{2} b_{1}+\frac{1}{3}\left(a_{1}-b_{1}\right)\right] \times\left[\frac{1}{2} b_{2}+\frac{1}{3}\left(a_{2}-b_{2}\right)\right] \times h_{A_{3}}\left[b_{3}+\left(a_{3}-b_{3}\right) h_{A 3}\right]\right\} d h_{\mathrm{A} 3} /\left(\frac{1}{2} \times \frac{1}{2} \times \int_{0}^{1} h_{\mathrm{A} 3} d_{\mathrm{A}_{3}}\right)$

$=\frac{1}{8}\left\{\left[\frac{1}{2} \mathrm{c}_{1}+\frac{1}{3}\left(\mathrm{a}_{1}-\mathrm{c}_{1}\right)\right] \times\left[\frac{1}{2} \mathrm{c}_{2}+\frac{1}{3}\left(\mathrm{a}_{2}-\mathrm{c}_{2}\right)\right] \times\left[\frac{1}{2} \mathrm{c}_{3}+\frac{1}{3}\left(\mathrm{a}_{3}-\mathrm{c}_{3}\right)\right]\right.$

$+\left[\frac{1}{2} b_{1}+\frac{1}{3}\left(a_{1}-b_{1}\right)\right] \times\left[\frac{1}{2} c_{2}+\frac{1}{3}\left(a_{2}-c_{2}\right)\right] \times\left[\frac{1}{2} c_{3}+\frac{1}{3}\left(a_{3}-c_{3}\right)\right]$

$+\left[\frac{1}{2} c_{1}+\frac{1}{3}\left(a_{1}-c_{1}\right)\right] \times\left[\frac{1}{2} b_{2}+\frac{1}{3}\left(a_{2}-b_{2}\right)\right] \times\left[\frac{1}{2} c_{3}+\frac{1}{3}\left(a_{3}-c_{3}\right)\right]$

$+\left[\frac{1}{2} c_{1}+\frac{1}{3}\left(a_{1}-c_{1}\right)\right] \times\left[\frac{1}{2} c_{2}+\frac{1}{3}\left(a_{2}-c_{2}\right)\right] \times\left[\frac{1}{2} b_{3}+\frac{1}{3}\left(a_{3}-b_{3}\right)\right]$

$+\left[\frac{1}{2} c_{1}+\frac{1}{3}\left(a_{1}-c_{1}\right)\right] \times\left[\frac{1}{2} b_{2}+\frac{1}{3}\left(a_{2}-b_{2}\right)\right] \times\left[\frac{1}{2} b_{3}+\frac{1}{3}\left(a_{3}-b_{3}\right)\right]$

$+\left[\frac{1}{2} b_{1}+\frac{1}{3}\left(a_{1}-b_{1}\right)\right] \times\left[\frac{1}{2} c_{2}+\frac{1}{3}\left(a_{2}-c_{2}\right)\right] \times\left[\frac{1}{2} b_{3}+\frac{1}{3}\left(a_{3}-b_{3}\right)\right]$

$+\left[\frac{1}{2} b_{1}+\frac{1}{3}\left(a_{1}-b_{1}\right)\right] \times\left[\frac{1}{2} b_{2}+\frac{1}{3}\left(a_{2}-b_{2}\right)\right] \times\left[\frac{1}{2} c_{3}+\frac{1}{3}\left(a_{3}-c_{3}\right)\right]$

$\left.+\left[\frac{1}{2} \mathrm{~b}_{1}+\frac{1}{3}\left(\mathrm{a}_{1}-\mathrm{b}_{1}\right)\right] \times\left[\frac{1}{2} \mathrm{~b}_{2}+\frac{1}{3}\left(\mathrm{a}_{2}-\mathrm{b}_{2}\right)\right] \times\left[\frac{1}{2} \mathrm{~b}_{3}+\frac{1}{3}\left(\mathrm{a}_{3}-\mathrm{b}_{3}\right)\right]\right\} \quad /\left(\frac{1}{2} \times \frac{1}{2} \times \frac{1}{2}\right)$

$=\left\{\left(\frac{c_{1}+2 a_{1}}{6}\right) \times\left(\frac{c_{2}+2 a_{2}}{6}\right) \times\left(\frac{c_{3}+2 a_{3}}{6}\right)+\left(\frac{b_{1}+2 a_{1}}{6}\right) \times\left(\frac{c_{2}+2 a_{2}}{6}\right) \times\left(\frac{c_{3}+2 a_{3}}{6}\right)\right.$

$+\left(\frac{\mathrm{c}_{1}+2 \mathrm{a}_{1}}{6}\right) \times\left(\frac{\mathrm{b}_{2}+2 \mathrm{a}_{2}}{6}\right) \times\left(\frac{\mathrm{c}_{3}+2 \mathrm{a}_{3}}{6}\right)+\left(\frac{\mathrm{c}_{1}+2 \mathrm{a}_{1}}{6}\right) \times\left(\frac{\mathrm{c}_{2}+2 \mathrm{a}_{2}}{6}\right) \times\left(\frac{\mathrm{b}_{3}+2 \mathrm{a}_{3}}{6}\right)$

$+\left(\frac{\mathrm{c}_{1}+2 \mathrm{a}_{1}}{6}\right) \times\left(\frac{\mathrm{b}_{2}+2 \mathrm{a}_{2}}{6}\right) \times\left(\frac{\mathrm{b}_{3}+2 \mathrm{a}_{3}}{6}\right)+\left(\frac{\mathrm{b}_{1}+2 \mathrm{a}_{1}}{6}\right) \times\left(\frac{\mathrm{c}_{2}+2 \mathrm{a}_{2}}{6}\right) \times\left(\frac{\mathrm{b}_{3}+2 \mathrm{a}_{3}}{6}\right)$

$\left.+\left(\frac{\mathrm{b}_{1}+2 \mathrm{a}_{1}}{6}\right) \times\left(\frac{\mathrm{b}_{2}+2 \mathrm{a}_{2}}{6}\right) \times\left(\frac{\mathrm{c}_{3}+2 \mathrm{a}_{3}}{6}\right)+\left(\frac{\mathrm{b}_{1}+2 \mathrm{a}_{1}}{6}\right) \times\left(\frac{\mathrm{b}_{2}+2 \mathrm{a}_{2}}{6}\right) \times\left(\frac{\mathrm{b}_{3}+2 \mathrm{a}_{3}}{6}\right)\right\}$

$=\left(\frac{c_{1}+2 a_{1}}{6}\right)\left(\frac{c_{2}+2 a_{2}}{6}\right)\left[\left(\frac{c_{3}+2 a_{3}}{6}\right)+\left(\frac{b_{3}+2 a_{3}}{6}\right)\right]$

$+\left(\frac{\mathrm{b}_{1}+2 \mathrm{a}_{1}}{6}\right)\left(\frac{\mathrm{c}_{2}+2 \mathrm{a}_{2}}{6}\right)\left[\left(\frac{\mathrm{c}_{3}+2 \mathrm{a}_{3}}{6}\right)+\left(\frac{\mathrm{b}_{3}+2 \mathrm{a}_{3}}{6}\right)\right]$

$+\left(\frac{\mathrm{c}_{1}+2 \mathrm{a}_{1}}{6}\right)\left(\frac{\mathrm{b}_{2}+2 \mathrm{a}_{2}}{6}\right)\left[\left(\frac{\mathrm{c}_{3}+2 \mathrm{a}_{3}}{6}\right)+\left(\frac{\mathrm{b}_{3}+2 \mathrm{a}_{3}}{6}\right)\right]$

$+\left(\frac{\mathrm{b}_{1}+2 \mathrm{a}_{1}}{6}\right)\left(\frac{\mathrm{b}_{2}+2 \mathrm{a}_{2}}{6}\right)\left[\left(\frac{\mathrm{c}_{3}+2 \mathrm{a}_{3}}{6}\right)+\left(\frac{\mathrm{b}_{3}+2 \mathrm{a}_{3}}{6}\right)\right]$

$=\left[\left(\frac{c_{1}+2 a_{1}}{6}\right)+\left(\frac{b_{1}+2 a_{1}}{6}\right) \times\left[\left(\frac{c_{2}+2 a_{2}}{6}\right)+\left(\frac{b_{2}+2 a_{2}}{6}\right) \times\left[\left(\frac{c_{3}+2 a_{3}}{6}\right)+\left(\frac{b_{3}+2 a_{3}}{6}\right)\right]\right.\right.$ 
$=\frac{1}{6}\left(c_{1}+4 a_{1}+b_{1}\right) \times \frac{1}{6}\left(c_{2}+4 a_{2}+b_{2}\right) \times \frac{1}{6}\left(c_{3}+4 a_{3}+b_{3}\right)$

We have that $P\left(A_{1} \otimes A_{2} \otimes A_{3}\right)=\frac{1}{6}\left(c_{1}+4 a_{1}+b_{1}\right) \times \frac{1}{6}\left(c_{2}+4 a_{2}+b_{2}\right) \times \frac{1}{6}\left(c_{3}+4 a_{3}+b_{3}\right)$

By formula (2), $\mathrm{P}\left(\mathrm{A}_{1}\right)=\frac{1}{6}\left(\mathrm{c}_{1}+4 \mathrm{a}_{1}+\mathrm{b}_{1}\right), \mathrm{P}\left(\mathrm{A}_{2}\right)=\frac{1}{6}\left(\mathrm{c}_{2}+4 \mathrm{a}_{2}+\mathrm{b}_{2}\right), \mathrm{P}\left(\mathrm{A}_{3}\right)=\frac{1}{6}\left(\mathrm{c}_{3}+4 \mathrm{a}_{3}+\mathrm{b}_{3}\right)$

$$
\mathrm{P}\left(\mathrm{A}_{1}\right) \otimes \mathrm{P}\left(\mathrm{A}_{2}\right) \otimes \mathrm{P}\left(\mathrm{A}_{3}\right)=\frac{1}{6}\left(\mathrm{c}_{1}+4 \mathrm{a}_{1}+\mathrm{b}_{1}\right) \times \frac{1}{6}\left(\mathrm{c}_{2}+4 \mathrm{a}_{2}+\mathrm{b}_{2}\right) \times \frac{1}{6}\left(\mathrm{c}_{3}+4 \mathrm{a}_{3}+\mathrm{b}_{3}\right)
$$

Thus, we have that $\mathrm{P}\left(\mathrm{A}_{1} \otimes \mathrm{A}_{2} \otimes \mathrm{A}_{3}\right)=\mathrm{P}\left(\mathrm{A}_{1}\right) \times \mathrm{P}\left(\mathrm{A}_{2}\right) \times \mathrm{P}\left(\mathrm{A}_{3}\right)$

\section{Application to the Container Distribution Center Location Selection}

The representation of multiplication operation on multiple triangular fuzzy numbers is applied to select the container distribution center location in China. This paper interviewed one Taiwanese shipping logistics company in China and then obtained the influential criteria, sub-criteria for container distribution center location selection. The influential criteria include the volume of containers aspect $\left(C_{1}\right)$, cost aspect $\left(C_{2}\right)$, land aspect $\left(C_{3}\right)$ and government policies aspect $\left(C_{4}\right)$. The sub-criteria of volume of containers aspect include the present volume of containers $\left(\mathrm{C}_{11}\right)$ and potential volume of containers in the future $\left(\mathrm{C}_{12}\right)$. The sub-criteria of cost aspect include the labor cost $\left(\mathrm{C}_{21}\right)$, transportation cost $\left(\mathrm{C}_{22}\right)$ and operation cost $\left(\mathrm{C}_{23}\right)$. The sub-criteria of land aspect include the availability of land $\left(\mathrm{C}_{31}\right)$, land cost $\left(\mathrm{C}_{32}\right)$, size of land $\left(\mathrm{C}_{33}\right)$ and distance from/to the city $\left(\mathrm{C}_{34}\right)$. The sub-criteria of government policies aspect include the efficiency of government department $\left(\mathrm{C}_{41}\right)$, co-operative relationship between the shipping logistics company and the government $\left(\mathrm{C}_{42}\right)$, tax break $\left(\mathrm{C}_{43}\right)$, other preferential treatment $\left(\mathrm{C}_{44}\right)$ and law on investment $\&$ investment restrictions $\left(\mathrm{C}_{45}\right)$. After preliminary screening, six candidate locations including the Shanghai $\left(\mathrm{K}_{1}\right)$, Qingdao $\left(\mathrm{K}_{2}\right)$, Ningbo $\left(\mathrm{K}_{3}\right)$, Tiangjin $\left(\mathrm{K}_{4}\right)$, Shenzhen $\left(\mathrm{K}_{5}\right)$ and Xiamen $\left(\mathrm{K}_{6}\right)$ remain for further evaluation. Besides, we also obtained the weights for criteria and sub-criteria from the Taiwanese shipping logistics company, including the weights $\mathrm{W}_{1}, \mathrm{~W}_{2}, \mathrm{~W}_{3}$, $\mathrm{W}_{4}$ for criteria $\mathrm{C}_{1}, \mathrm{C}_{2}, \mathrm{C}_{3}, \mathrm{C}_{4}$ respectively, and the weights $\mathrm{W}_{11}, \mathrm{~W}_{12}, \mathrm{~W}_{21}, \mathrm{~W}_{22}, \mathrm{~W}_{23}$, $\mathrm{W}_{31}, \mathrm{~W}_{32}, \mathrm{~W}_{33}, \mathrm{~W}_{34}, \mathrm{~W}_{41}, \mathrm{~W}_{42}, \mathrm{~W}_{43}, \mathrm{~W}_{44}, \mathrm{~W}_{45}$ for sub-criteria $\mathrm{C}_{11}, \mathrm{C}_{12}, \mathrm{C}_{21}, \mathrm{C}_{22}, \mathrm{C}_{23}$, $\mathrm{C}_{31}, \mathrm{C}_{32}, \mathrm{C}_{33}, \mathrm{C}_{34}, \mathrm{C}_{41}, \mathrm{C}_{42}, \mathrm{C}_{43}, \mathrm{C}_{44}, \mathrm{C}_{45}$ respectively. Similarly, we also obtained the performance $\left(\mathrm{P}_{\mathrm{ijk}}\right)$ for candidate location $\mathrm{K}$ under each criteria $\mathrm{i}$ and sub-criteria $\mathrm{j}$. The data of the weight for criteria and the weight for sub-criteria for the selection of container distribution center location are listed as follows.

Criteria Weights:

$\mathrm{W}_{1}=(0.3,0.35,0.4), \quad \mathrm{W}_{2}=(0.15,0.2,0.25), \quad \mathrm{W}_{3}=(0.15,0.2,0.25)$ and $\mathrm{W}_{4}=(0.2,0.25,0.3)$ Sub-Criteria Weights:

$\mathrm{W}_{11}=(0.6,0.7,0.8), \mathrm{W}_{12}=(0.2,0.3,0.4), \mathrm{W}_{21}=(0.2,0.3,0.4), \mathrm{W}_{22}=(0.3,0.4,0.5), \mathrm{W}_{23}=(0.2,0.3,0.4)$ $\mathrm{W}_{31}=(0.2,0.3,0.4), \mathrm{W}_{32}=(0.15,0.2,0.25), \mathrm{W}_{33}=(0.15,02,025), \mathrm{W}_{34}=(0.2,0.3,0.4), \mathrm{W}_{41}=(0.15,0.2,0.25)$, $\mathrm{W}_{42}=(0.15,0.2,0.25), \mathrm{W}_{43}=(0.15,0.2,0.25), \mathrm{W}_{44}=(0.15,0.2,0.25)$ and $\mathrm{W}_{45}=(0.15,0.2,0.25)$. 


\section{Solution Process}

Total Performance of candidate location $\mathrm{K}_{1}: \mathrm{TP}_{1}$

$$
\mathrm{TP}_{1}=\sum_{1}^{I} \sum_{1}^{J} \mathrm{~W}_{\mathrm{i}} \otimes \mathrm{W}_{\mathrm{ij}} \otimes \mathrm{P}_{\mathrm{ij} 1}
$$

$=\mathrm{W}_{1} \otimes \mathrm{W}_{11} \otimes \mathrm{P}_{111}+\mathrm{W}_{1} \otimes \mathrm{W}_{12} \otimes \mathrm{P}_{121}+\mathrm{W}_{2} \otimes \mathrm{W}_{21} \otimes \mathrm{P}_{211}+\mathrm{W}_{2} \otimes \mathrm{W}_{22} \otimes \mathrm{P}_{221}+$ $\mathrm{W}_{2} \otimes \mathrm{W}_{23} \otimes \mathrm{P}_{231}+\mathrm{W}_{3} \otimes \mathrm{W}_{31} \otimes \mathrm{P}_{311}+\mathrm{W}_{3} \otimes \mathrm{W}_{32} \otimes \mathrm{P}_{321}+\mathrm{W}_{3} \otimes \mathrm{W}_{33} \otimes \mathrm{P}_{331}+$ $\mathrm{W}_{3} \otimes \mathrm{W}_{34} \otimes \mathrm{P}_{341}+\mathrm{W}_{4} \otimes \mathrm{W}_{41} \otimes \mathrm{P}_{411}+\mathrm{W}_{4} \otimes \mathrm{W}_{42} \otimes \mathrm{P}_{421}+\mathrm{W}_{4} \otimes \mathrm{W}_{43} \otimes \mathrm{P}_{431}+$ $\mathrm{W}_{4} \otimes \mathrm{W}_{44} \otimes \mathrm{P}_{441}+\mathrm{W}_{4} \otimes \mathrm{W}_{45} \otimes \mathrm{P}_{451}$

$$
\begin{aligned}
& =(0.3,0.35,0.4) \otimes(0.6,0.7,0.8) \otimes(10,10,10)+(0.3,0.35,0.4) \otimes(0.2,0.3,0.4) \otimes(7,8,9) \\
& +(0.15,0.2,0.25) \otimes(0.2,0.3,0.4) \otimes(6,7,8)+(0.15,0.2,0.25) \otimes(0.3,0.4,0.5) \otimes(7,8,9) \\
& +(0.15,0.2,0.25) \otimes(0.2,0.3,0.4) \otimes(6,7,8)+(0.15,0.2,0.25) \otimes(0.2,0.3,0.4) \otimes(8,9,10) \\
& +(0.15,0.2,0.25) \otimes(0.15,0.2,0.25) \otimes(6,7,8)+(0.15,0.2,0.25) \otimes(0.15,0.2,0.25) \otimes(7,8,9) \\
& +(0.15,0.2,0.25) \otimes(0.2,0.3,0.4) \otimes(10,10,10)+(0.2,0.25,0.3) \otimes(0.15,0.2,0.25) \otimes(8,9,10) \\
& +(0.2,0.25,0.3) \otimes(0.15,0.2,0.25) \otimes(7,8,9)+(0.2,0.25,0.3) \otimes(0.15,0.2,0.25) \otimes(7,8,9) \\
& +(0.2,0.25,0.3) \otimes(0.15,0.2,0.25) \otimes(7,8,9)+(0.2,0.25,0.3) \otimes(0.15,0.2,0.25) \otimes(7,8,9) \\
& =8.56
\end{aligned}
$$

By the same solution process, we can obtain the total performance of candidate location $\mathrm{TP}_{2}=7.87, \mathrm{TP}_{3}=7.53, \mathrm{TP}_{4}=6.98, \mathrm{TP}_{5}=5.84, \mathrm{TP}_{6}=5.71$. The candidate locations Shanghai $\left(\mathrm{K}_{1}\right)$, Qingdao $\left(\mathrm{K}_{2}\right)$ and Ningbo $\left(\mathrm{K}_{3}\right)$ are selected as the container distribution center locations by the Taiwanese shipping logistics company in China.

\section{Conclusion}

This paper proposes the representation of multiplication operation on multiple fuzzy numbers and this representation is applied to the decision making model for Taiwanese shipping logistics company in China to select the container distribution center location under fuzzy multiple criteria decision making environment. Based on this representation, the decision maker of Taiwanese shipping logistics company in China can rank quickly the ordering of candidate location and then choose easily the best one. This representation of multiplication operation on multiple fuzzy numbers proposed in this paper not only can be applied to solve the location selection problem of container distribution center, but also can be applied to other fuzzy multiple criteria decision making problems.

On the other hand, we can see while selecting one container distribution center the Taiwanese shipping logistics company is much care about the present volume of containers, potential volume of containers in the future, labor cost, transportation cost, operation cost, availability of land, land cost, size of land, distance from/to the city, efficiency of government department, co-operative relationship between the shipping logistics company and the government, tax break, other preferential treatment, and law on investment \& investment restrictions. Thus this paper suggests that the 
departments of government and the managers of ports should pay attention to the above 14 criteria to maintain the advantages and to improve the disadvantages.

\section{References}

1. Bellman, R.E., Zadeh, L.A.: Decision-making in a Fuzzy Environment. Management Science 17(4), 141-164 (1970)

2. Chen, C.T.: A Fuzzy Approach to Select the Location of the Distribution Center. Fuzzy Sets and Systems 118(1), 65-73 (2001)

3. Chen, S.H., Hsieh, C.H.: Graded Mean Integration Representation of Generalized Fuzzy Number. In: Proceedings of 6th Conference on Fuzzy Theory and Its Application (CDROM), Filename: 031.wdl, Chinese Fuzzy Systems Association, Taiwan, P.R. China, pp. 1-6 (1998)

4. Chou, C.C.: The Canonical Representation of Multiplication Operation on Triangular Fuzzy Numbers. Computers \& Mathematics with Applications 45, 1601-1610 (2003)

5. Chou, C.C.: Representation of Multiplication Operation on Fuzzy Numbers and Application to Solving Fuzzy Multiple Criteria Decision Making Problems. In: Yang, Q., Webb, G. (eds.) PRICAI 2006. LNCS (LNAI), vol. 4099, pp. 161-169. Springer, Heidelberg (2006)

6. Chou, C.C.: A Fuzzy MCDM Method for Solving Marine Transshipment Container Port Selection Problems. Applied Mathematics and Computation 186, 435-444 (2007)

7. Dahlberg, M.D., May, J.H.: Linear Programming for Sitting of Energy Facilities. Journal of Energy Engineering, 5-14 (1980)

8. Delgado, M., Vila, M.A., Voxman, W.: On a Canonical Representation of Fuzzy Numbers. Fuzzy Sets and Systems 93, 125-135 (1998)

9. Dubois, D., Prade, H.: Operations on Fuzzy Numbers. Journal of Systems Sciences 9, 613$626(1978)$

10. Hsu, H.M., Chen, C.T.: Fuzzy Credibility Relation Method for Multiple Criteria Decisionmaking Problems. Information Sciences 96, 79-91 (1997)

11. Hwang, C.L., Yoon, K.: Multiple Attributes Decision Making Methods and Applications. Springer, Berlin, Heidelberg (1981)

12. Li, R.J.: Fuzzy Method in Group Decision Making. Computers and Mathematics with Applications 38, 91-101 (1999)

13. Liang, G.S., Wang, M.J.: A Fuzzy Multiple Criteria Decision-making Method for Facilities Site Selection. International Journal of Production Research 29(11), 2313-2330 (1991)

14. Ma, M., Friedman, M., Kandel, A.: A New Fuzzy Arithmetic. Fuzzy Sets and Systems 108, 83-90 (1999)

15. Mizumoto, M., Tanaka, K.: The Four Operations of Arithmetic on Fuzzy Numbers. Systems Comput. Controls 7(5), 73-81 (1976)

16. Nahmias, S.: Fuzzy Variables. Fuzzy Sets and Systems, 97-111 (1978)

17. Rietveld, P., Ouwersloot, H.: Ordinal Data in Multi-criteria Decision Making, a Stochastic Dominance Approach to Sitting Nuclear Power Plants. European Journal of Operation Research 56, 249-262 (1992)

18. Spohrer, G.A., Kmak, T.R.: Qualitative Analysis Used in Evaluating Alternative Plant Location Scenarios. Industrial Engineering 16, 52-56 (1984)

19. Stevenson, W. J.: Production/ Operation Management. Richard D. Irwin Inc. Illinois (1993) 
20. Tompkins, J.A., White, J.A.: Facilities Planning. John Wiley \& Sons Company, New York (1984)

21. Yong, D.: Plant Location Selection Based on Fuzzy TOPSIS. International Journal of Advanced Manufacturing Technology 28, 839-844 (2006)

22. Yong, D., Cheng, S.: Evaluating the Main Battle Tank Using Fuzzy Number Arithmetic Operations. Defence Science Journal 56, 251-257 (2006)

23. Zadeh, L.A.: Fuzzy Sets. Information and Control 8, 338-353 (1965)

24. Zadeh, L.A.: The Concept of a Linguistic Variable and Its Application to Approximate Reasoning. Information Sciences 8, 199-249 (1975) 